\title{
Controllable synthesis of band-gap-tunable and monolayer transition-metal dichalcogenide alloys
}

\author{
Sheng-Han Su ${ }^{1,2}$, Wei-Ting Hsu ${ }^{3}$, Chang-Lung Hsu ${ }^{1}$, Chang-Hsiao Chen ${ }^{1}$, Ming-Hui Chiu ${ }^{1}$, Yung-Chang Lin ${ }^{4}$, \\ Wen-Hao Chang ${ }^{3}$, Kazu Suenaga ${ }^{4}$, Jr-Hau He ${ }^{2}$ and Lain-Jong $\mathrm{Li}^{1}{ }^{*}$
}

1 Institute of Atomic and Molecular Sciences, Academia Sinica, Taipei, Taiwan

${ }^{2}$ Department of Electrical Engineering, Graduate Institute of Photonics and Optoelectronics, National Taiwan University, Taipei, Taiwan

${ }^{3}$ Department of Electrophysics, National Chiao Tung University, Hsinchu, Taiwan

${ }^{4}$ National Institute of Advanced Industrial Science and Technology (AIST), Tsukuba, Japan

\section{Edited by:}

Peng Chen, Nanyang Technological

University, Singapore

Reviewed by:

Xiaochen Dong, Nanjing Tech

University, China

Hongchang Pang, Dalian University of Technology, China

Jianwen Zhao, Chinese Academy of Sciences, China

\section{*Correspondence}

Lain-Jong Li, Institute of Atomic and Molecular Sciences, Academia Sinica, P.O. Box 23-166 Taipei, 10617, Taiwan e-mail: lanceli@gate.sinica.edu.tw

\begin{abstract}
The electronic and optical properties of transition-metal dichalcogenide (TMD) materials are directly governed by their energy gap; thus, band-gap engineering has become an important topic recently. Theoretical and some experimental results have indicated that these monolayerTMD alloys exhibit direct-gap properties and remain stable at room temperature, making them attractive for optoelectronic applications. Here, we systematically compared the two approaches of forming $\mathrm{MoS}_{2 x} \mathrm{Se}_{2(1-x)}$ monolayer alloys: selenization of $\mathrm{MoS}_{2}$ and sulfurization of $\mathrm{MoSe}_{2}$. The optical energy gap of as-grown chemical vapor deposition $\mathrm{MoS}_{2}$ can be continuously modulated from $1.86 \mathrm{eV}(667 \mathrm{~nm})$ to $1.57 \mathrm{eV}(790 \mathrm{~nm})$ controllable by the reaction temperature. Spectroscopic and microscopic evidences show that the Mo-S bonds can be replaced by the Mo-Se bonds in a random and homogeneous manner. By contrast, the replacement of Mo-Se by Mo-S does not randomly occur in the $\mathrm{MoSe}_{2}$ lattice, where the reaction preferentially occurs along the crystalline orientation of $\mathrm{MoSe}_{2}$ and thus the $\mathrm{MoSe}_{2} / \mathrm{MoS}_{2}$ biphases are easily observed in the alloys, which makes the optical band gap of these alloys distinctly different. Therefore, the selenization of metal disulfide is preferred and the proposed synthetic strategy opens up a simple route to control the atomic structure as well as optical properties of monolayer TMD alloys.
\end{abstract}

Keywords: transition-metal dichalcogenides, $\mathrm{MoS}_{2}, \mathrm{MoSe}_{2}, \mathrm{WS}_{2}, \mathrm{WS} \mathrm{e}_{2}$, band-gap tuning, layered materials, two-dimensional materials

\section{INTRODUCTION}

Two-dimensional transition-metal dichalcogenide (TMD) monolayers quickly emerge due to their promising properties, including moderate carrier mobility values (Radisavljevic et al., 2011; Kaasbjerg et al., 2012), good bendability, and direct band gaps (Mak et al., 2010; Splendiani et al., 2010; Wang et al., 2012). These advantages could be beneficial to many applications such as low-power electronics (Radisavljevic et al., 2011; Yu et al., 2012; Lin et al., 2013), flexible applications (Pu et al., 2012, 2014), optoelectronic devices (Bernardi et al., 2013; Oriol et al., 2013; Tsai et al., 2013; Zhang et al., 2013; Hsu et al., 2014), and catalysis (Chang et al., 2014). Since the optical responsivity and conversion efficiency at different wavelengths in optoelectronic devices are dominated by the optical band gap of the TMD monolayer, band-gap engineering has become one of the research focuses. It has been reported that the optical band gap of TMD monolayers can be modified by strain engineering (Feng et al., 2012; Lu et al., 2012; Pan and Zhang, 2012; Peelaers and Van de Walle, 2012; Hui et al., 2013) or the formation of heterojunction TMD layers (Ghorbani-Asl et al., 2013). Alternatively, monolayer TMD alloys such as $\mathrm{Mo}_{\mathrm{x}} \mathrm{W}_{1-\mathrm{x}} \mathrm{S}_{2}$ have been obtained by mechanical exfoliation from the corresponding TMD solid solutions (Chen et al., 2013). The recent breakthrough in the chemical vapor deposition (CVD) growth of $\mathrm{MoS}_{2}, \mathrm{WSe}_{2}$, and $\mathrm{WS}_{2}$ monolayers (Lee et al., 2012, 2013; Huang et al., 2014) has stimulated the direct growth of monolayer TMD alloys. Several recent manuscripts have shown that $\mathrm{MoS}_{2 \mathrm{x}} \mathrm{Se}_{2(1-\mathrm{x})}$ is obtainable through the gas phase reaction of $\mathrm{MoO}_{3}$ with the mixture of $\mathrm{S}$ and Se (Gong et al., 2013; Li et al., 2014; Mann et al., 2014). These preliminary works prove the theoretical prediction that TMD monolayer alloys are stable at room temperature (Jiang, 2012). However, large variations in atomic composition from flakes to flakes are expected owing to the difficulty to precisely control the $\mathrm{S}$ and Se vapor diffusion in a CVD furnace. A scalable method to synthesize monolayer TMD alloys with controllable optical energy gaps is still urgently needed for practical applications.

In this contribution, we show that both the CVD-grown molybdenum and tungsten dichalcogenides monolayer flakes can be selenized or sulfurized at high temperatures. The optical band gap of the obtained $\mathrm{MoS}_{\mathrm{x}} \mathrm{Se}_{\mathrm{y}}$ and $\mathrm{WS}_{\mathrm{x}} \mathrm{Se}_{\mathrm{y}}$ monolayers ranges from 1.57 to $2.0 \mathrm{eV}$. Spectroscopic and microscopic results suggest that the replacement of Mo-S by Mo-Se occurs more homogeneously compared to the replacement of Mo-Se by Mo-S. It is suggested that the selenization of metal disulfides is more favorable in the synthetic control of the compositions and optical properties for monolayer TMD alloys.

\section{MATERIALS AND METHODS SYNTHESIS OF MONOLAYER TMD}

The CVD-grown $\mathrm{MoS}_{2}, \mathrm{MoSe}_{2}, \mathrm{WS}_{2}$, and WSe $\mathrm{W}_{2}$ monolayers were synthesized based on several previous reports (Lee et al., 2012, 


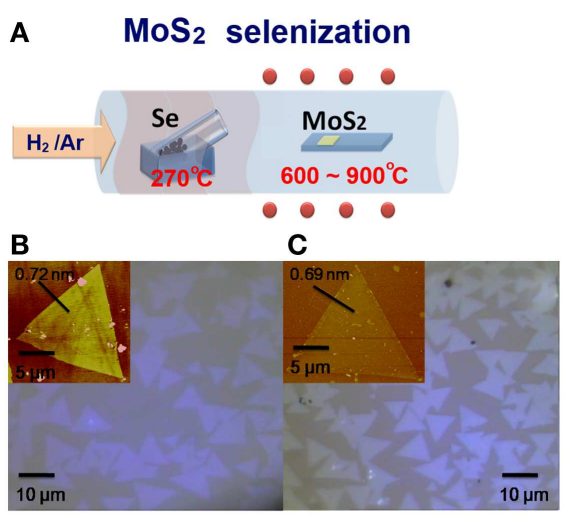

FIGURE 1 | (A,D) Schematic illustration of the experimental set-up for the selenization/sulfurization process, where the inlet gas carries the vaporized selenium (sulfur) to the heated $\mathrm{MoS}_{2}\left(\mathrm{MoSe}_{2}\right)$ flakes and optical micrographs for the (B,E) as-synthesized $\mathrm{MoS}_{2} / \mathrm{MoSe}_{2}$, and

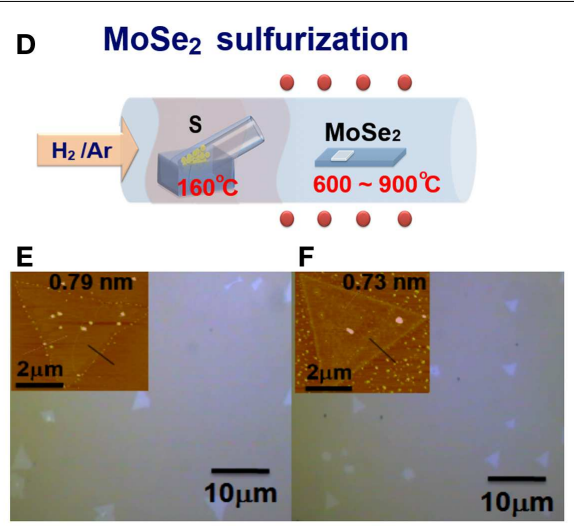

(C,F) selenized (sulfurized) $\mathrm{MoS}_{2} / \mathrm{MoSe}_{2}$ (at $800^{\circ} \mathrm{C}$ ) on sapphire substrates. AFM images for the $\mathrm{MoS}_{2} / \mathrm{MoSe}_{2}$ flakes before and after selenization (sulfurization) (at $800^{\circ} \mathrm{C}$ ) are shown as insets in $\mathrm{OM}$ images.
2013; Lin et al., 2012; Huang et al., 2014). Taking monolayer $\mathrm{MoS}_{2}$ single crystals as an example, $c$-plane sapphire (0001) substrates (Tera Xtal Technology Corp.) were first cleaned in a piranha solution $\left[\mathrm{H}_{2} \mathrm{SO}_{4} / \mathrm{H}_{2} \mathrm{O}_{2}(70: 30)\right]$ at $100^{\circ} \mathrm{C}$ for $1 \mathrm{~h}$. Substrates were then placed in the center of a $4^{\prime \prime}$ tubular furnace on a quartz holder. The $\mathrm{MoO}_{3}$ powders ( $0.6 \mathrm{~g}$; Sigma-Aldrich, $99.5 \%$ ) in an $\mathrm{Al}_{2} \mathrm{O}_{3}$ crucible were placed next to the sapphire substrates. The S (Sigma-Aldrich, 99.5\%) powders were placed at the upstream position, where the schematic illustration of the growth system was described elsewhere (Zhang et al., 2013). The furnace was first heated to $150^{\circ} \mathrm{C}$ at $10^{\circ} \mathrm{C} / \mathrm{min}$ rate with $70 \mathrm{sccm}$ Ar at 10 torr and annealed for $20 \mathrm{~min}$, then ramped to $650^{\circ} \mathrm{C}$ at $25^{\circ} \mathrm{C} / \mathrm{min}$ rate and kept for $20 \mathrm{~min}$. Sulfur was heated separately by heating belt to $170^{\circ} \mathrm{C}$ when the furnace reached $400^{\circ} \mathrm{C}$. After growth, furnace was slowly cooled to room temperature.

\section{SELENIZATION (SULFURIZATION) PROCESS}

The as-grown monolayer $\mathrm{MoS}_{2}\left(\mathrm{MoSe}_{2}\right)$ and $\mathrm{WS}_{2}\left(\mathrm{WSe}_{2}\right)$ single crystal flakes were selenized (sulfurized) in a hot-wall furnace at $600,700,800$, and $900^{\circ} \mathrm{C}$. Briefly, as-grown $\mathrm{MoS}_{2}\left(\mathrm{MoSe}_{2}\right)$ and $\mathrm{WS}_{2}\left(\mathrm{WSe}_{2}\right)$ monolayers on sapphire were placed in the center of the quartz tube. The selenium (sulfur) powders were placed at the upstream position and heated to $270^{\circ} \mathrm{C}\left(160^{\circ} \mathrm{C}\right)$ with a separate heating belt. The furnace was heated to $600,700,800$, and $900^{\circ} \mathrm{C}$ at $30^{\circ} \mathrm{C} / \mathrm{min}$ rate and kept for $2 \mathrm{~h}$ for reaction. After selenization (sulfurization), the furnace was slowly cooled to room temperature.

\section{CHARACTERIZATIONS}

Photoluminescence spectra were performed with a green light $(532 \mathrm{~nm})$ laser and 0.9 NA of objective lens (spot size: $0.7 \mu \mathrm{m}$ ). Raman spectra were collected in a NT-MDT confocal Raman microscopic system (laser wavelength $473 \mathrm{~nm}$ and laser spot size $\sim 0.5 \mu \mathrm{m})$. The Si peak, $520 \mathrm{~cm}^{-1}$, was used as reference for wavenumber calibration. The atomic force microscopy (AFM) images were performed in a Veeco Dimension-Icon system. The transmittance spectra of the $\mathrm{MoS}_{2}$ flakes were obtained using a
JASCO-V-670 UV-Vis spectrophotometer. Chemical configurations were determined by X-ray photoelectron spectroscope (XPS, Phi V5000). XPS measurements were performed with an $\mathrm{Mg} \mathrm{K} \alpha$ $\mathrm{X}$-ray source on the samples. The energy calibrations were made against the $\mathrm{C} 1 \mathrm{~s}$ peak to eliminate the charging of the sample during analysis.

\section{RESULTS AND DISCUSSIONS}

As described in the Section "Materials and Methods," the triangular monolayer TMD flakes with a lateral size up to several tens of microns can be obtained by our proposed CVD method and it has been adopted by many other groups (Amani et al., 2013; Zhao et al., 2013). In order to tune the electronic structures and optical band gaps of TMD monolayers, we perform the selenization/sulfurization in a hot-wall furnace at various temperatures. The schemes in Figures 1A,D illustrate the experimental set-up for the selenization and sulfurization processes, respectively, where the inlet gas (a mixture of $\mathrm{Ar}$ and $\mathrm{H}_{2}$ ) carries the vaporized selenium or sulfur to the heated $\mathrm{MoS}_{2}$ or $\mathrm{MoSe}_{2}$ flakes. It should be noted that hydrogen gas is required in the process to avoid the oxidation of $\mathrm{MoS}_{2}$ or $\mathrm{MoSe}_{2}$ by residual oxygen or unavoidable oxygen leaking from the environment. Figures 1B,E show the optical micrographs for the as-synthesized $\mathrm{MoS}_{2}$ and $\mathrm{MoSe}_{2}$ on sapphire substrates before selenization or sulfurization. Those for the selenized $\mathrm{MoS}_{2}$ and sulfurized $\mathrm{MoSe}_{2}$ at $800^{\circ} \mathrm{C}$ are displayed in Figures 1C,F, respectively. There is no obvious change in size and shape of the $\mathrm{MoS}_{2}\left(\mathrm{MoSe}_{2}\right)$ flakes after selenization (sulfurization). The AFM image for the $\mathrm{MoS}_{2}\left(\mathrm{MoSe}_{2}\right)$ flakes before and after selenization (sulfurization) at $800^{\circ} \mathrm{C}$ is shown in inset of each OM image. Note that the slight changes in thickness after selenization or sulfurization are within the AFM measurement errors.

The Raman spectra for the $\mathrm{MoS}_{2}\left(\mathrm{MoSe}_{2}\right)$ flakes before and after selenization (sulfurization) at different temperatures are shown in Figure 2. No significant changes are observed for the $\mathrm{MoS}_{2}\left(\mathrm{MoSe}_{2}\right)$ samples after selenization (sulfurization) at $600^{\circ} \mathrm{C}$, where the $\mathrm{MoS}_{2}\left(\mathrm{MoSe}_{2}\right)$ characteristic peaks, 385.6 (286.3) 


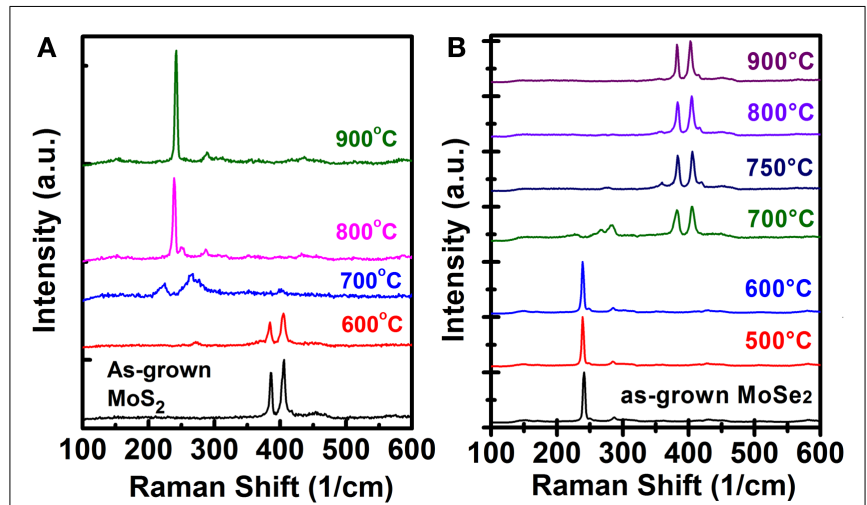

FIGURE 2 | Raman spectra for the (A) $\mathrm{MoS}_{2}$ and (B) $\mathrm{MoSe}_{2}$ flakes before and after selenization/sulfurization at different temperatures are shown

$\mathrm{cm}^{-1}$ for $\mathrm{E}_{2 \mathrm{~g}}^{1}$ mode and $405.8(241.1) \mathrm{cm}^{-1}$ for $\mathrm{A}_{1 \mathrm{~g}}$ mode, are predominant in both as-grown and $600^{\circ} \mathrm{C}$-reacted samples. As the temperature increases to $700^{\circ} \mathrm{C}$, both $\mathrm{MoS}_{2}$ and $\mathrm{MoSe}_{2}$ flakes after respective selenization and sulfurization exhibit several unidentified peaks at around $200-300 \mathrm{~cm}^{-1}$, which are likely attributed to the vibration from the partially selenized Mo-S or sulfurized Mo-Se structures and worth further investigations in the future. When the process is performed at $800^{\circ} \mathrm{C}\left(750^{\circ} \mathrm{C}\right), \mathrm{MoS}_{2}\left(\mathrm{MoSe}_{2}\right)$ flakes start to be converted to $\mathrm{MoSe}_{2}\left(\mathrm{MoS}_{2}\right)$, which is evidenced by the presence of the Raman $E_{2 g}^{1}\left(A_{1 g}\right)$ and $A_{1 g}\left(E_{2 g}^{1}\right)$ modes of $\mathrm{MoSe}_{2}\left(\mathrm{MoS}_{2}\right)$ at $287.1(383.1) \mathrm{cm}^{-1}$ and $239.0(405.4) \mathrm{cm}^{-1}$, respectively (Tonndorf et al., 2013; Su et al., 2014). It should be mentioned that in Figure 2A, the weak shoulder and unassigned peak occurred at around $249.8 \mathrm{~cm}^{-1}$ for the selenization process, which implies that the quality of $\mathrm{MoSe}_{2}$ is still not perfect. When the selenized temperature of $\mathrm{MoS}_{2}$ reaches $900^{\circ} \mathrm{C}$, the perfect $\mathrm{A}_{1 \mathrm{~g}}$ and $E_{2 g}^{1}$ peaks are shown at 242.2 and $289 \mathrm{~cm}^{-1}$. Meanwhile, the shoulder peak completely disappears and no $\mathrm{MoS}_{2}$ feature peaks are observed, indicating the selenization process is successful. On the other hand, the $\mathrm{MoSe}_{2}$ flakes sulfurized at 800 and $900^{\circ} \mathrm{C}$ exhibit two sharp $\mathrm{MoS}_{2}$ Raman peaks. Hence, we could confirm that both $\mathrm{MoS}_{2}$ and $\mathrm{MoSe}_{2}$ can be successfully converted to each other at a higher process temperature.

$\mathrm{X}$-ray photoemission spectroscopy was utilized to characterize the chemical bonding structures of these monolayer flakes. Figure 3 displays the detailed XPS scans for the Mo, S, and Se binding energies for the as-grown $\mathrm{MoS}_{2}\left(\mathrm{MoSe}_{2}\right)$ and those after selenization (sulfurization), where the magnitude of each profile was normalized for easier comparison. From Figure 3A, the peaks at 232.5 and $229.3 \mathrm{eV}$ represent the Mo $3 \mathrm{~d}_{3 / 2}$ and Mo $3 \mathrm{~d}_{5 / 2}$ binding energies for $\mathrm{Mo}^{4+}$ (Majumdera and Takoudis, 2008). The $\mathrm{S}$ $2 \mathrm{p}_{1 / 2}$ and $\mathrm{S} 2 \mathrm{p}_{3 / 2}$ orbital of divalent sulfide ions $\left(\mathrm{S}^{2-}\right)$ are identified at 163.3 and $162.1 \mathrm{eV}$ (Wang et al., 2013). Moreover, the observation of a doublet peak $\left(232.4\right.$ and $\left.235.6 \mathrm{~cm}^{-1}\right)$ indicates the presence of $\mathrm{MoO}_{3}$. For selenization process, a weak doublet peak of Se $3 \mathrm{~d}_{3 / 2}\left(55.3 \mathrm{~cm}^{-1}\right)$ and Se $3 \mathrm{~d}_{5 / 2}\left(54.5 \mathrm{~cm}^{-1}\right)$ binding energy appears even after a low temperature process $\left(600^{\circ} \mathrm{C}\right)$, which means that XPS is more sensitive than Raman in the structural characterization for monolayer alloys. As the selenization temperature increases, the doublet peak of $\mathrm{Se} 3 \mathrm{~d}_{3 / 2}$, Se $3 \mathrm{~d}_{5 / 2}$ becomes more dominant and the binding energy peak of $S 2 p_{1 / 2}$ and $S 2 p_{3 / 2}$ gradually disappears. After $900^{\circ} \mathrm{C}$ selenization, the $\mathrm{S}$ characteristic peaks are absent, further confirming that the $\mathrm{MoS}_{2}$ selenization process is successfully completed. Similarly, the results for $\mathrm{MoSe}_{2}$ sulfurization shown in the Figure 3B demonstrate that $\mathrm{MoSe}_{2}$ can be sulfurized to $\mathrm{MoS}_{2}$ at $900^{\circ} \mathrm{C}$. Both the selenization and sulfurization processes take effects at the temperature higher than $600^{\circ} \mathrm{C}$.

We perform the photoluminescence measurements using microscopically focused light $(532 \mathrm{~nm}$; spot size: $0.7 \mu \mathrm{m})$ to study the optical properties of selenized $\mathrm{MoS}_{2}$. Figure 4A shows the photoluminescence spectra collected for the samples before and after selenization at different temperatures. The emission peak wavelength $667 \mathrm{~nm}$ for the $\mathrm{MoS}_{2}$ selenized at $600^{\circ} \mathrm{C}$ is still pretty similar to the $668 \mathrm{~nm}$ peak of the pristine $\mathrm{MoS}_{2}$ samples. The peak wavelength for the sample selenized at $700^{\circ} \mathrm{C}$ is at $726 \mathrm{~nm}$ and the wavelength continues to increase to 768 and $790 \mathrm{~nm}$ for the samples selenized at 800 and $900^{\circ} \mathrm{C}$, respectively. The emission wavelength $(790 \mathrm{~nm})$ for the $\mathrm{MoS}_{2}$ selenized at $900^{\circ} \mathrm{C}$ is very close to the reported values from $792 \mathrm{~nm}$ (Tonndorf et al., 2013) to $800 \mathrm{~nm}$ (Kong et al., 2013) for the exfoliated monolayer $\mathrm{MoSe}_{2}$. Figure 4B shows the optical absorption spectrum for these samples. Two distinct absorption peaks at approximately 794.2 and $700.4 \mathrm{~nm}$, identified as A and B excitonic absorptions are observed and these peak positions are consistent with those for exfoliated $\mathrm{MoSe}_{2}$ (Tonndorf et al., 2013). It should be mentioned that $\mathrm{A}$ and $\mathrm{B}$ excitons are generated by the splitting of valence band due to the spin-orbital splitting effect. It is obvious that $\mathrm{MoS}_{2}$ gradually transforms into $\mathrm{MoSe}_{2}$ from 600 to $900^{\circ} \mathrm{C}$. By considering the photoluminescence, absorption spectra, and Raman features, it is suggested that the $900^{\circ} \mathrm{C}$ selenized sample is close to that of the reported $\mathrm{MoSe}_{2}$ monolayer (Tonndorf et al., 2013). Moreover, we present in Figures 4C,D the energy variation and the spin-orbital splitting vs. selenized temperature. The optical energy gap gradually becomes lower with the temperature, consistent with the PL results. From the absorption peaks, the spin-orbital splitting can be derived as $180 \mathrm{meV}$ for $\mathrm{MoS}_{2}$ and $250 \mathrm{meV}$ for $\mathrm{MoSe}_{2}$. The optical features in Figure 4 are in line with the calculations by Komsa and Krasheninnikov (2012) where the entropic contributions promote the mixing of $\mathrm{MoS}_{2}$ and $\mathrm{MoSe}_{2}$ (Jiang, 2012), which is energetically more preferable over the segregated phases in the mixture even at $300 \mathrm{~K}$. The optical properties of $\mathrm{MoSe}_{2}$ before and after sulfurization are shown in the Figure 5. The emission peak at $804 \mathrm{~nm}$ for $\mathrm{MoSe}_{2}$ sulfurized by $600^{\circ} \mathrm{C}$ is also similar to that of the as-grown $\mathrm{MoSe}_{2}$. The PL peak remains at $809 \mathrm{~nm}$ but becomes broader after sulfurization at $700^{\circ} \mathrm{C}$, which suggests that the $\mathrm{MoSe}_{2}$ starts to react with $\mathrm{S}$ at around $700^{\circ} \mathrm{C}$. In clear contrast to the selenization of $\mathrm{MoS}_{2}$, Figures 5A,B show that the PL and absorption features of the $\mathrm{MoSe}_{2}$ suddenly changes to $\mathrm{MoS}_{2}$-like when the sulfurization temperature is equal to or higher than $750^{\circ} \mathrm{C}$. The optical band gap of the sulfurized $\mathrm{MoSe}_{2}$ does not seem to be adjustable as those obtained from the selenization of $\mathrm{MoS}_{2}$. The spin-orbital 

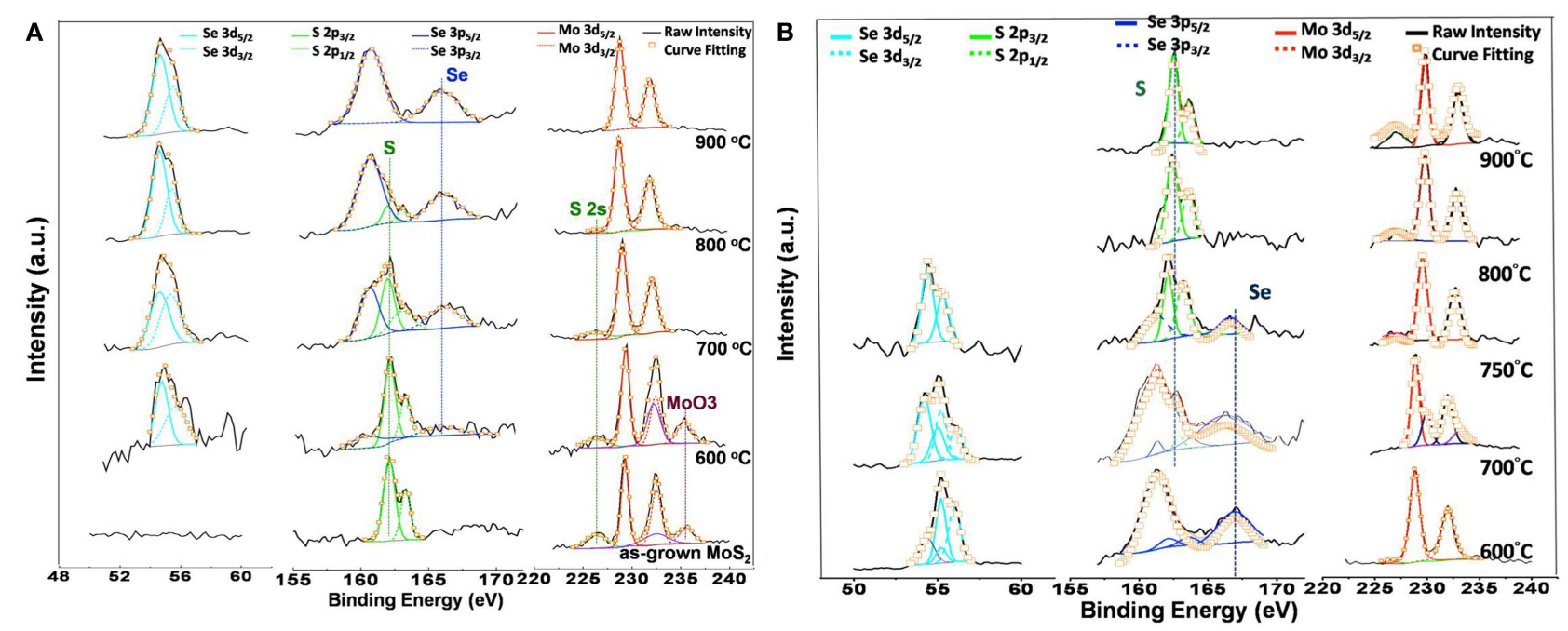

FIGURE 3 | XPS scans for the Mo, S, and Se binding energies for (A) the as-grown $\mathrm{MoS}_{2}$ and those after selenization, and (B) the as-grown MoSe ${ }_{2}$ and those after sulfurization. The magnitude of each profile was normalized for easier comparison.

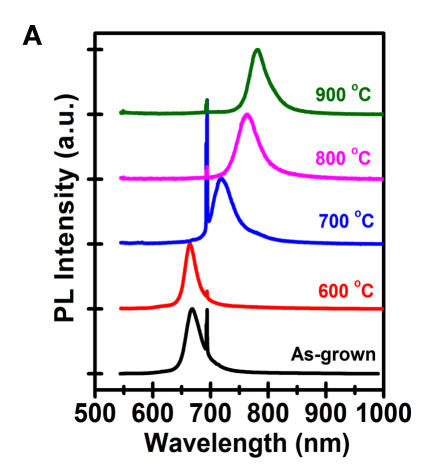

C
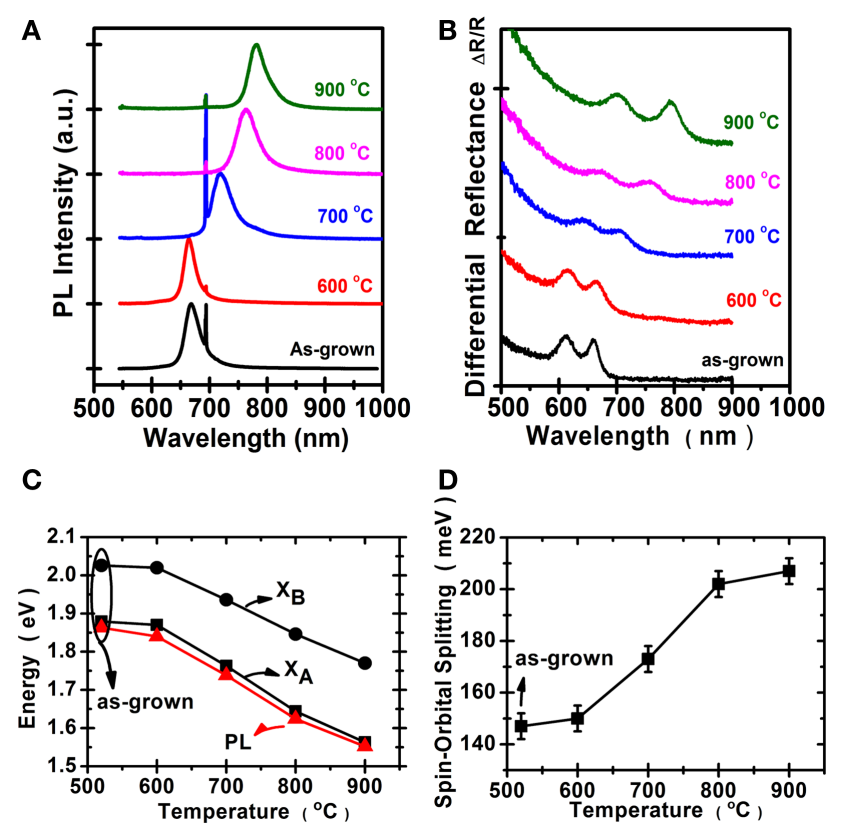

FIGURE 4 | Optical properties of $\mathrm{MoS}_{2}$ selenization are collected for the samples before and after selenization: (A) Photoluminescence spectra, the peak at $694 \mathrm{~nm}$ was from the sapphire substrate.

(B) Absorption spectra. (C) The optical energy gap of (A,B), exciton, and PL peaks. (D) The spin-orbital splitting derived from the absorption spectra.

splitting energy with the sulfurization temperature also shows the same behavior.

In order to microscopically reveal the homogeneity of the samples, some flakes are selected for visualization under TEM. Figures 6A,B are the $\mathrm{MoS}_{2}$ flakes after selenization at 600 and $700^{\circ} \mathrm{C}$, respectively. Both Figures demonstrate that the

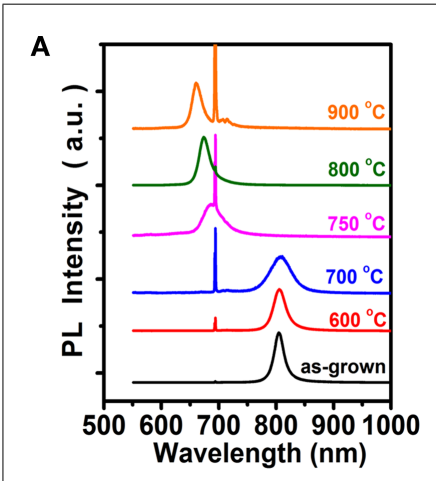

C
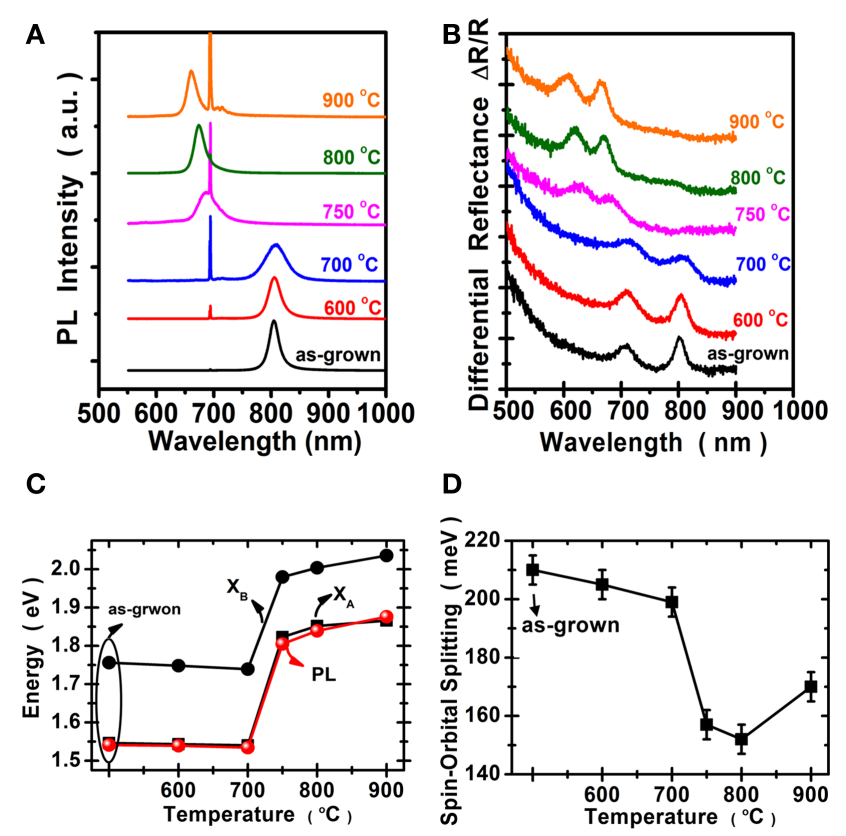

FIGURE 5 | Optical properties of the $\mathrm{MoSe}_{2}$ are collected before and after sulfurization. (A) Photoluminescence spectra, where the peak at $694 \mathrm{~nm}$ was from the sapphire substrate. (B) Absorption spectra. (C) The optical energy gap of (A,B), excitons, and PL peaks. (D) The spin-orbital splitting derived from the absorption spectra.

replacement of S by Se is random and homogeneous in the lattice. It is noted that some replacement occurs at $600^{\circ} \mathrm{C}$ although the Raman, PL, and absorption spectra do not show pronounced changes as described before. After the $\mathrm{MoS}_{2}$ is selenized at $700^{\circ} \mathrm{C}$, large numbers of $\mathrm{S}_{2}, \mathrm{Se}_{2}$, and $\mathrm{S}-\mathrm{Se}$ are identified and the replacement seems to be not only randomly located but also with some 

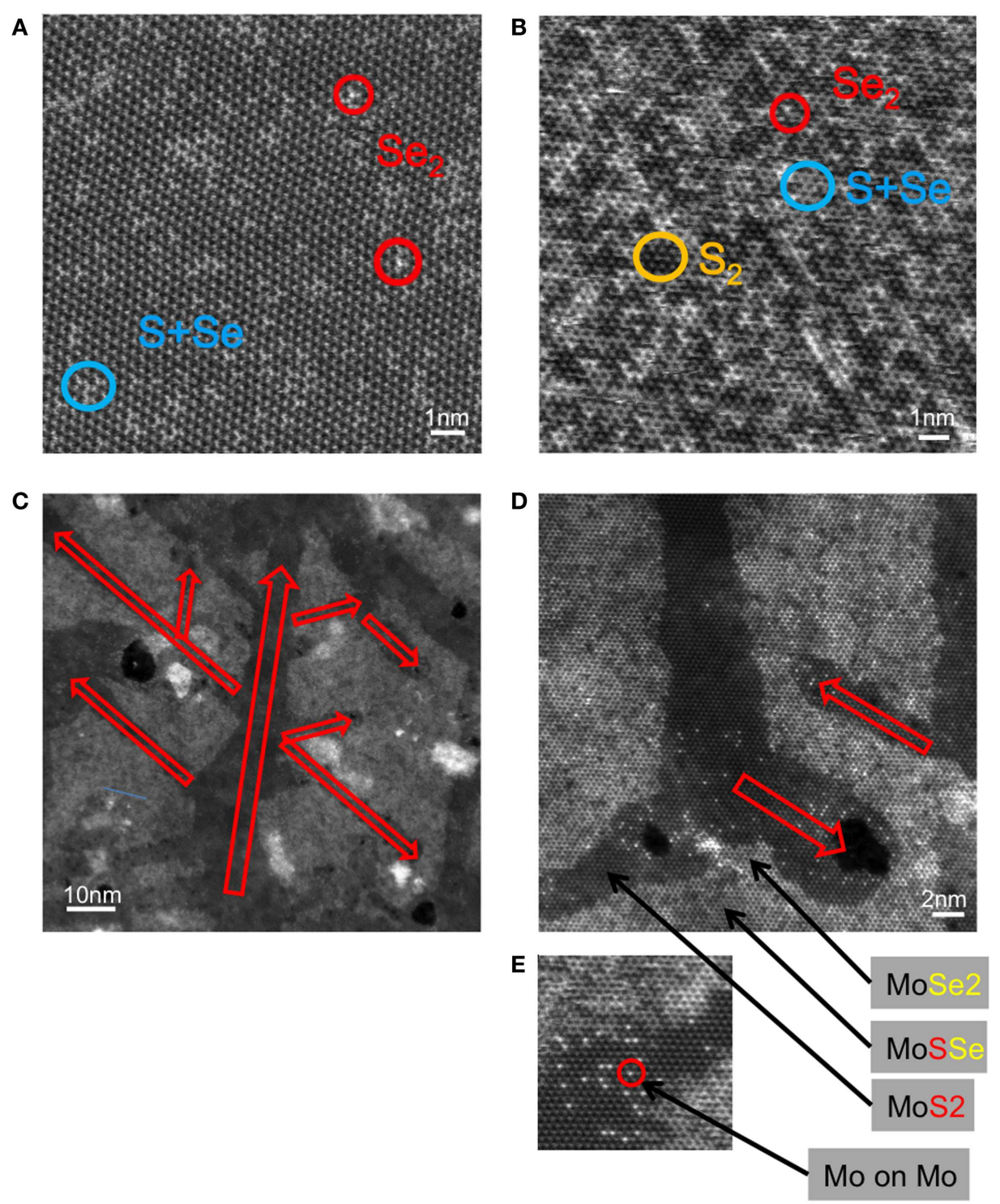

FIGURE 6 |TEM images of the $\mathrm{MoS}_{2}$ flakes selenized at (A) $600^{\circ} \mathrm{C}$ and (B) $700^{\circ} \mathrm{C}$ and (C-E) $\mathrm{MoSe}_{2}$ sulfurized at $700^{\circ} \mathrm{C}$

identifiable triangle patterns. Since it is generally homogeneous in location, the optical properties can be considered to originate from the $\mathrm{MoS}_{\mathrm{x}} \mathrm{Se}_{\mathrm{y}}$ alloy. By contrast, Figures $6 \mathrm{C}-\mathrm{E}$ show the TEM images for the $\mathrm{MoSe}_{2}$ flakes after sulfurization at $700^{\circ} \mathrm{C}$. The field is composed of two obvious $\mathrm{MoS}_{2}$ and $\mathrm{MoSe}_{2}$ domains, where MoSSe domains are also found in some areas. In general, the sulfurization seems to preferentially proceed through certain directions, which are related to the crystal orientation. Since theoretical prediction suggests that the mixing of $\mathrm{MoS}_{2}$ and $\mathrm{MoSe}_{2}$ is energetically more preferable over the segregated phases, the formation of biphases is likely due to the kinetic factor. Meanwhile, the preferential formation of biphases in morphology can also explain their distinctly different optical properties, where the $\mathrm{PL}$ and absorption peaks are either dominated by $\mathrm{MoS}_{2}$ or $\mathrm{MoSe}_{2}$, depending on the degree of sulfurization.
In addition to molybdenum dichalcogenides, the same method has also been utilized to selenize (sulfurize) the $\mathrm{WS}_{2}\left(\mathrm{WSe}_{2}\right)$ flakes, respectively. Figures 7B,C show the Raman and PL spectra for the $\mathrm{WS}_{2}$ flakes after selenization at various temperatures. Similar to the case of $\mathrm{MoS}_{2}$ selenization, we can see the gradual increase in emission wavelength from 621 to $684 \mathrm{~nm}$ when the selenization temperature increases from 600 to $850^{\circ} \mathrm{C}$. However, when the temperature reaches $900^{\circ} \mathrm{C}$, all the triangular flakes are completely gone, which could be observed in Figure 7A. Hence, it could infer that the thermostability of $\mathrm{WS}_{2}$ is not as good as $\mathrm{MoS}_{2}$. The consistent results are revealed in the Raman spectra in Figure 8B, where the $\mathrm{WS}_{2}$ peaks at 358 and $419 \mathrm{~cm}^{-1}$ gradually disappear and the $\mathrm{WSe}_{2}$ peak at $279 \mathrm{~cm}^{-1}$ becomes pronounced with increasing temperature. On the flip side, $\mathrm{WSe}_{2}$, similar to $\mathrm{MoSe}_{2}$, could be fully and successfully 

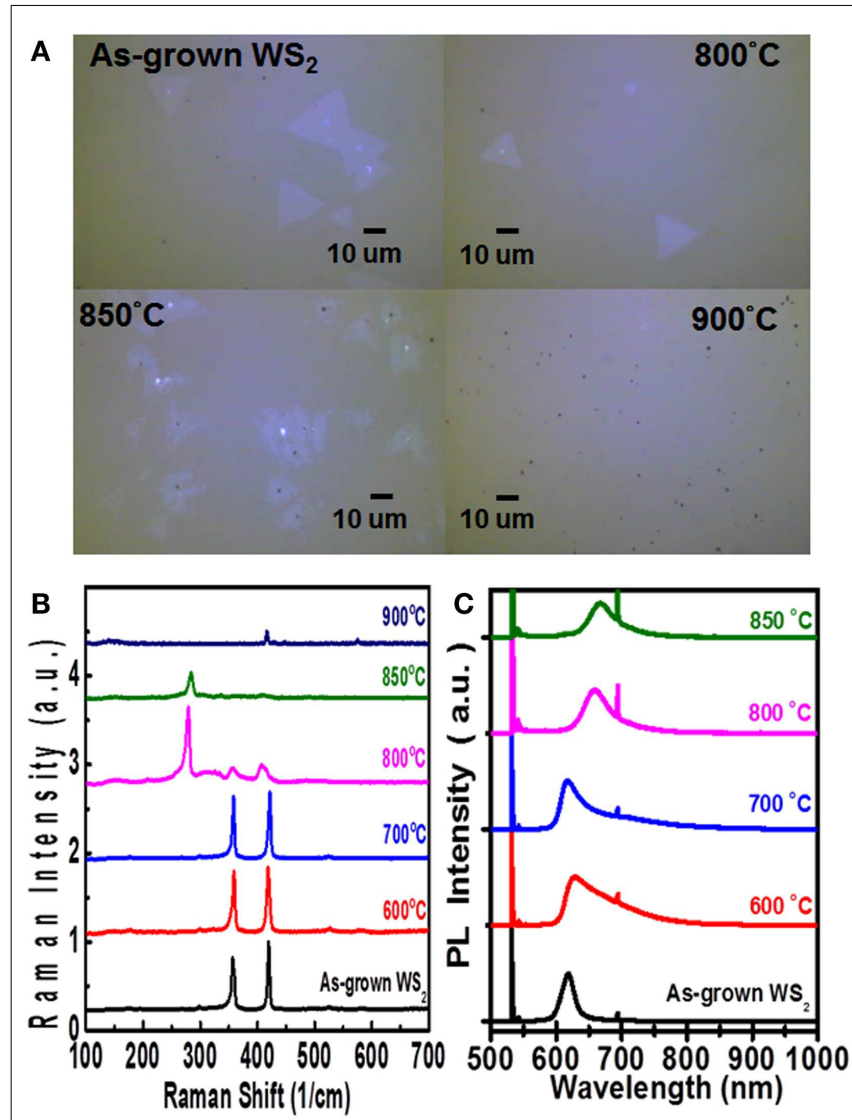

FIGURE 7 | (A) Optical images, (B) Raman spectra, and (C) PL spectra for the $\mathrm{WS}_{2}$ monolayer flakes after selenization at various temperatures are shown.

sulfurized. Figure 8A displays the AFM and OM images for the $\mathrm{WSe}_{2}$ flakes before and after sulfurization at $800^{\circ} \mathrm{C}$. The Raman spectra in Figure 8B reveal that the $\mathrm{WSe}_{2}$ peaks located at 250 and $260 \mathrm{~cm}^{-1}$ still remain at $700^{\circ} \mathrm{C}$. When the temperature reaches $800^{\circ} \mathrm{C}$, the Raman peaks from $\mathrm{WS}_{2}$ are dominated, but PL peak is still closer to $\mathrm{WSe}_{2}$ emission. The PL results in Figure $\mathbf{8 C}$ suggest that the sample is composed of biphase domains, similar to the previously described sulfurization of $\mathrm{MoS}_{2}$. These suggest that $\mathrm{WSe}_{2}$ starts to but is not yet completely transformed to $\mathrm{WS}_{2}$. Based on the PL results, the sulfurization of $\mathrm{WSe}_{2}$ starts to become pronounced when the temperature is higher than $900^{\circ} \mathrm{C}$.

To reveal the chemical composition, we estimate the $\mathrm{S}$ content in Figure 9 for each sample by the ratio between Mo-S and (Mo-Se $+\mathrm{Mo}-\mathrm{S}$ ) based on XPS spectra (not shown here). For example, the Mo-S percentage for the as-grown $\mathrm{MoS}_{2}$ and those selenized at $600,700,800$, and $900^{\circ} \mathrm{C}$ is $100,85.8,26.2$, 5 , and $0 \%$, respectively. The observed trend strongly agrees the conversion of Mo-S to Mo-Se bonds. The solid line represents the experimental results by Gong et al. (2013) and the dash line is the theoretical results (Kang et al., 2013). It is clearly seen
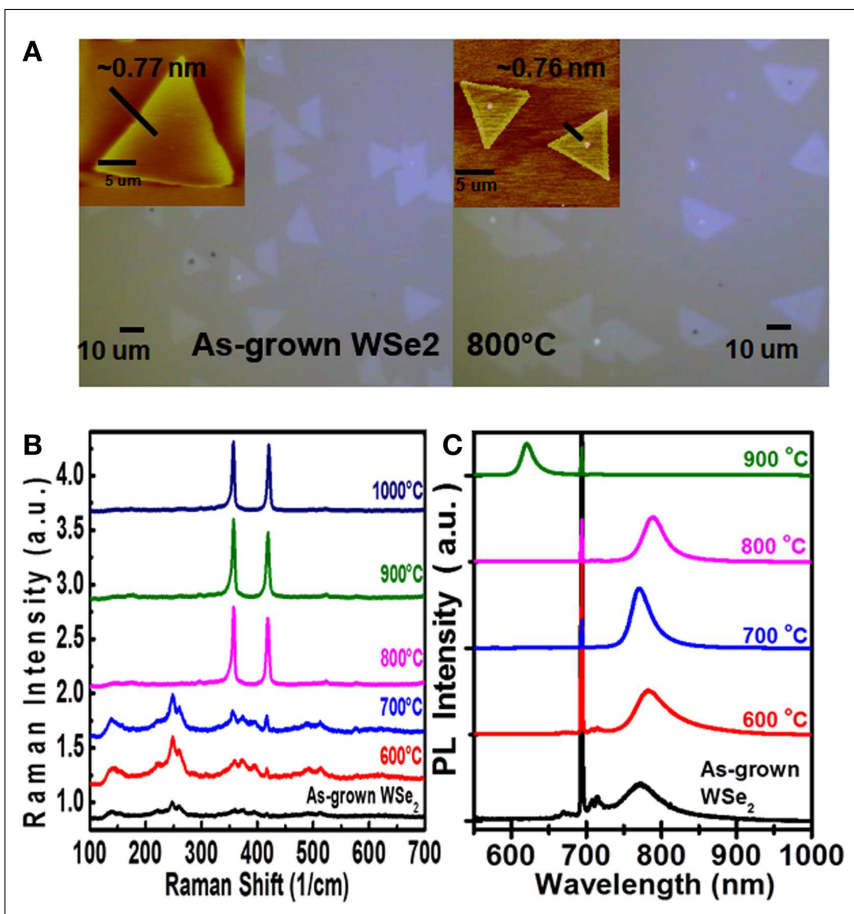

FIGURE 8 | (A) OM and AFM images, (B) Raman spectra, and (C) PL spectra of the $\mathrm{WSe}_{2}$ flakes after sulfurization at various temperatures.

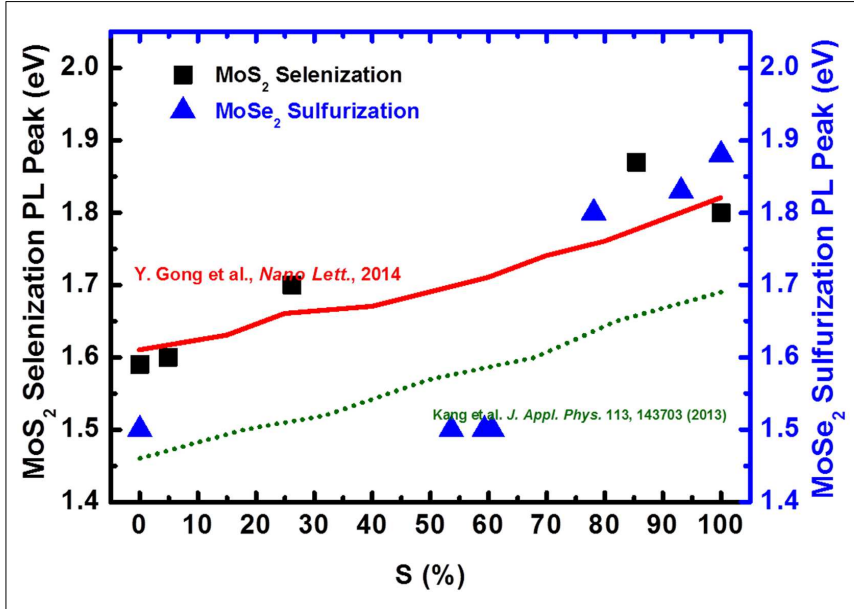

FIGURE 9 |The optical gap of selenization (square symbol) and sulfurization (triangle symbol) with the variation of Sulfur composition is shown. The experimental from Gong et al. (solid line) and theoretical results (dash line) are for references.

that the experimental optical band gap is larger than the calculated gap. Most importantly, the conversion is governed by the selenization temperature, indicating that the process is thermodynamically controlled. However, due to the presence of biphase domains, the PL peak position vs. $\mathrm{S} \%$ for $\mathrm{MoSe}_{2}$ sulfurization exhibits distinctly different behavior compared to that for $\mathrm{MoS}_{2}$ selenization. 


\section{CONCLUSION}

In summary, we report that the CVD-grown TMD monolayer flakes can be successfully selenized and sulfurized with a simple and straightforward method. The optical band gap, ranging from $1.57 \mathrm{eV}(790 \mathrm{~nm})$ to $2.0 \mathrm{eV}(620 \mathrm{~nm})$, is controllable by the selenization or sulfurization temperatures. TEM analysis indicates that the Mo-S bonds can be replaced by the Mo-Se bonds in a random and homogeneous manner. By contrast, the replacement of Mo-Se by Mo-S does not randomly occur in the $\mathrm{MoSe}_{2}$ lattice, leading to the biphase formation.

\section{ACKNOWLEDGMENTS}

This research was mainly supported by Academia Sinica (IAMS and Nano program) and National Science Council Taiwan (NSC102-2119-M-001-005-MY3).

\section{REFERENCES}

Amani, M., Chin, M. L., Birdwell, A. G., O’Regan, T. P., Najmaei, S., Liu, Z., et al. (2013). Electrical performance of monolayer $\mathrm{MoS}_{2}$ field-effect transistors prepared by chemical vapor deposition. Appl. Phys. Lett. 102, 193107. doi:10.1063/1.4804546

Bernardi, M., Palummo, M., and Grossman, J. C. (2013). Extraordinary sunlight absorption and one nanometer thick photovoltaics using two-dimensional monolayer materials. Nano Lett. 13, 3664-3670. doi:10.1021/nl401544y

Chang, Y.-H., Wu, F.-Y., Chen, T.-Y., Hsu, C.-L., Chen, C.-H., Wiryo, F., et al. (2014). Three-dimensional molybdenum sulfide sponges for electrocatalytic water splitting. Small 5, 895-900. doi:10.1002/smll.201302407

Chen, Y., Xi, J., Dumcenco, D. O., Liu, Z., Suenaga, K., Wang, D., et al. (2013). Tunable band gap photoluminescence from atomically thin transition-metal dichalcogenide alloys. ACS Nano 5, 4610-4616. doi:10.1021/nn401420h

Feng, J., Qian, X., Huang, C.-W., and Li, J. (2012). Strain-engineered artificial atom as a broad-spectrum solar energy funnel. Nat. Photon 6, 866-872. doi: 10.1038/nphoton.2012.285

Ghorbani-Asl, M., Borini, S., Kuc, A., and Heine, T. (2013). Strain-dependent modulation of conductivity in single-layer transition-metal dichalcogenides. Phys. Rev. B 87, 235434. doi:10.1103/PhysRevB.87.235434

Gong, Y., Liu, Z., Lupini, A. R., Shi, G., Lin, J., Najmaei, S., et al. (2013). Band gap engineering and layer-by-layer mapping of selenium-doped molybdenum disulfide. Nano Lett. 14, 442-449. doi:10.1021/nl4032296

Hsu, W.-T., Zhao, Z.-A., Li, L.-J., Chen, C.-H., Chiu, M.-H., Chang, P.-S., et al. (2014). Second harmonic generation from artificially stacked transition metal dichalcogenide twisted bilayers. ACS Nano 8, 2951-2958. doi:10.1021/ nn500228r

Huang, J.-K., Pu, J., Hsu, C.-L., Chiu, M.-H., Juang, Z.-Y., Chang, Y.-H., et al. (2014). Large-area synthesis of highly crystalline $\mathrm{WSe}_{2}$ monolayers and device applications. ACS Nano 8, 923-930. doi:10.1021/nn405719X

Hui, Y. Y., Liu, X., Jie, W., Chan, N. Y., Hao, J., Hsu, Y.-T., et al. (2013). Exceptional tunability of band energy in a compressively strained trilayer $\mathrm{MoS}_{2}$ sheet. ACS Nano 7, 7126-7131. doi:10.1021/nn4024834

Jiang, H. (2012). Electronic band structures of molybdenum and tungsten dichalcogenides by the GW approach. J. Phys. Chem. C 116, 7664-7671. doi:10.1021/ jp300079d

Kaasbjerg, K., Thygesen, K. S., and Jacobsen, K. W. (2012). Phonon-limited mobility in n-type single-layer $\mathrm{MoS}_{2}$ from first principles. Phys. Rev. B 85, 115317. doi:10.1103/PhysRevB.85.115317

Kang, J., Tongay, S., Li, J., and Wu, J. (2013). Monolayer semiconducting transition metal dichalcogenide alloys: stability and band bowing. J. Appl. Phys. 113, 143703. doi:10.1063/1.4799126

Komsa, H.-P., and Krasheninnikov, V. A. (2012). Two-dimensional transition metal dichalcogenide alloys: stability and electronic properties. J. Phys. Chem. Lett. 3, 3652-3656. doi:10.1021/jz301673x

Kong, D., Wang, H., Cha, J. J., Pasta, M., Koski, J. K., Yao, J., et al. (2013). Synthesis of $\mathrm{MoS}_{2}$ and $\mathrm{MoSe}_{2}$ films with vertically aligned layers. Nano Lett. 13, 1341-1347. doi: $10.1021 / \mathrm{nl} 400258 \mathrm{t}$
Lee, Y.-H., Yu, L., Wang, H., Fang, W., Ling, X., Shi, Y., et al. (2013). Synthesis and transfer of single-layer transition metal disulfides on diverse surfaces. Nano Lett. 13, 1852-1857. doi:10.1021/nl400687n

Lee, Y.-H., Zhang, X.-Q., Zhang, W., Chang, M.-T., Lin, C.-T., Chang, K.-D., et al. (2012). Synthesis of large-area $\mathrm{MoS}_{2}$ atomic layers with chemical vapor deposition. Adv. Mater. 24, 2320-2325. doi:10.1002/adma.201104798

Li, H., Duan, X., Wu, X., Zhuang, X., Zhou, H., Zhang, Q., et al. (2014). Growth of alloy $\mathrm{MoS}_{2 \mathrm{x}} \mathrm{Se}_{2(1-\mathrm{x})}$ nanosheets with fully tunable chemical compositions and optical properties. J. Am. Chem. Soc. 136, 3756-3759. doi:10.1021/ ja500069b

Lin, J., Zhong, J., Zhong, S., Li, H., Zhang, H., and Chen, W. (2013). Modulating electronic transport properties of MoS2 field effect transistor by surface overlayers. Appl. Phys. Lett. 103, 063109. doi:10.1063/1.4818463

Lin, Y.-C., Zhang, W., Huang, J.-K., Liu, K.-K., Lee, Y.-H., Liang, C.-T., et al. (2012). Wafer-scale $\mathrm{MoS}_{2}$ thin layers prepared by $\mathrm{MoO}_{3}$ sulfurization. Nanoscale 4, 6637. doi:10.1039/c2nr31833d

Lu, P., Wu, X., Guo, W., and Zeng, X. C. (2012). Strain-dependent electronic and magnetic properties of $\mathrm{MoS}_{2}$ monolayer, bilayer, nanoribbons and nanotubes. Phys. Chem. Chem. Phys. 14, 13035-13040. doi:10.1039/c2cp42181j

Majumdera, P., and Takoudis, C. (2008). Reactively sputtered Mo-V nitride thin films as ternary diffusion barriers for copper metallization. J. Electrochem. Soc. 155, H703-H706. doi:10.1149/1.2955726

Mak, K. F., Lee, C., Hone, J., Shan, J., and Heinz, T. F. (2010). Atomically thin $\mathrm{MoS}_{2}$ : a new direct-gap semiconductor. Phys. Rev. Lett. 105, 136805. doi:10.1103/PhysRevLett.105.136805

Mann, J., Ma, Q., Odenthal, P. M., Isarraraz, M., Le, D., Preciado, E., et al. (2014). 2-Dimensional transition metal dichalcogenides with tunable direct band gaps: $\operatorname{MoS}_{2(1-x)}$ Se $_{2 x}$ monolayers. Adv. Mater. 26, 1399-1404. doi:10.1002/adma. 201304389

Oriol, L.-S., Dominik, L., Metin, K., Aleksandra, R., and Andras, K. (2013). Ultrasensitive photodetectors based on monolayer $\mathrm{MoS}_{2}$. Nat. Nanotechnol. 8, 497-501. doi:10.1038/nnano.2013.100

Pan, H., and Zhang, Y.-W. (2012). Tuning the electronic and magnetic properties of $\mathrm{MoS}_{2}$ nanoribbons by strain engineering. J. Phys. Chem. C 116, 11752-11757. doi:10.1021/jp3015782

Peelaers, H., and Van de Walle, C. G. (2012). Effects of strain on band structure and effective masses in $\mathrm{MoS}_{2}$. Phys. Rev. B 86, 241401(R). doi:10.1103/PhysRevB.86. 241401

Pu, J., Li, L.-J., and Takenobu, T. (2014). Flexible and stretchable thin-film transistors based on molybdenum disulphide. Phys. Chem. Chem. Phys. 16, 14996-15006. doi:10.1039/c3cp55270e

Pu, J., Yomogida, Y., Liu, K.-K., Li, L.-J., Iwasa, Y., and Takenobu, T. (2012). Highly flexible $\mathrm{MoS}_{2}$ thin-film transistors with ion gel dielectrics. Nano Lett. 12, 4013-4017. doi:10.1021/nl301335q

Radisavljevic, B., Radenovic, A., Brivio, J., Giacometti, V., and Kis, A. (2011). Singlelayer $\mathrm{MoS}_{2}$ transistors. Nat. Nanotechnol. 6, 147-150. doi:10.1038/nnano.2010. 279

Splendiani, A., Sun, L., Zhang, Y., Li, T., Kim, J., Chim, C.-Y., et al. (2010). Emerging photoluminescence in monolayer $\mathrm{MoS}_{2}$. Nano Lett. 10, 1271-1275. doi:10.1021/nl903868w

Su, S.-H., Hsu, Y.-T., Chang, Y.-H., Chiu, M.-H., Hsu, C.-L., Hsu, W.-T., et al. (2014). Band gap-tunable molybdenum sulfide selenide monolayer alloy. Small doi:10.1002/smll.201302893

Tonndorf, P., Schmidt, R., Böttger, P., Zhang, X., Börner, J., Liebig, A., et al. (2013). Photoluminescence emission and Raman response of monolayer $\mathrm{MoS}_{2}, \mathrm{MoSe}_{2}$, and WSe ${ }_{2}$. Opt. Exp. 21, 4908-4916. doi:10.1364/OE.21.004908

Tsai, D.-S., Liu, K.-K., Lien, D.-H., Tsai, M.-L., Kang, C.-F., Lin, C.-A., et al. (2013). Few-layer $\mathrm{MoS}_{2}$ with high broadband photogain and fast optical switching for use in harsh environments. ACS Nano 7, 3905-3911. doi:10.1021/ nn305301b

Wang, H., Yu, L., Lee, Y.-H., Shi, Y., Hsu, A., Chin, M. L., et al. (2012). Integrated circuits based on bilayer $\mathrm{MoS}_{2}$ transistors. Nano Lett. 12, 4674-4680. doi: $10.1021 / \mathrm{nl} 302015 \mathrm{v}$

Wang, X., Feng, H., Wu, Y., and Jiao, L. (2013). Controlled synthesis of highly crystalline $\mathrm{MoS}_{2}$ flakes by chemical vapor deposition. J. Am. Chem. Soc. 135, 5304-5307. doi:10.1021/ja4013485

Yu, W. J., Li, Z., Zhou, H., Chen, Y., Wang, Y., Huang, Y., et al. (2012). Vertically stacked multi-heterostructures of layered materials for logic transistors and complementary inverters. Nat. Mater. 12, 246-252. doi:10.1038/nmat3518 
Zhang, W., Chuu, C.-P., Huang, J.-K., Chen, C.-H., Tsai, M.-L., Chang, Y.-H., et al. (2013). Ultrahigh-gain photodetectors based on atomically thin graphene- $\mathrm{MoS}_{2}$ heterostructures. Sci. Rep. 4, 3826. doi:10.1038/srep03826

Zhao, Y., Luo, X., Li, H., Zhang, J., Araujo, T. P., Gan, C. K., et al. (2013). Interlayer breathing and shear modes in few-trilayer $\mathrm{MoS}_{2}$ and $\mathrm{WSe}_{2}$. Nano Lett. 13, 1007-1015. doi:10.1021/nl304169w

Conflict of Interest Statement: The authors declare that the research was conducted in the absence of any commercial or financial relationships that could be construed as a potential conflict of interest.

Received: 12 June 2014; paper pending published: 18 June 2014; accepted: 23 June 2014; published online: 08 July 2014.
Citation: Su S-H, Hsu W-T, Hsu C-L, Chen C-H, Chiu M-H, Lin Y-C, Chang W-H, Suenaga $\mathrm{K}, \mathrm{He} J-H$ and $\mathrm{Li}$ L-J (2014) Controllable synthesis of band-gap-tunable and monolayer transition-metal dichalcogenide alloys. Front. Energy Res. 2:27. doi: 10.3389/fenrg.2014.00027

This article was submitted to Nanoenergy Technologies and Materials, a section of the journal Frontiers in Energy Research.

Copyright (C) 2014 Su, Hsu, Hsu, Chen, Chiu, Lin, Chang, Suenaga, He and Li. This is an open-access article distributed under the terms of the Creative Commons Attribution License (CC BY). The use, distribution or reproduction in other forums is permitted, provided the original author(s) or licensor are credited and that the original publication in this journal is cited, in accordance with accepted academic practice. No use, distribution or reproduction is permitted which does not comply with these terms. 